\title{
Update on the epidemiology of gastro-oesophageal reflux disease: a systematic review
}

\author{
Hashem B El-Serag ${ }^{1}$, Stephen Sweet ${ }^{2}$, Christopher C Winchester ${ }^{2,3}$, and John Dent ${ }^{4}$ \\ ${ }^{1}$ Section of Gastroenterology and Hepatology, Houston Veterans Affairs Medical Center (152), \\ Houston, Texas, USA \\ ${ }^{2}$ Research Evaluation Unit, Oxford PharmaGenesis, Oxford, UK \\ ${ }^{3}$ Centre of Academic Primary Care, University of Aberdeen, Aberdeen, UK \\ ${ }^{4}$ Department of Gastroenterology, Hepatology and General Medicine, Royal Adelaide Hospital, \\ Adelaide, South Australia, Australia
}

\begin{abstract}
Objective-To update the findings of the 2005 systematic review of population-based studies assessing the epidemiology of gastro-oesophageal reflux disease (GERD).
\end{abstract}

\begin{abstract}
Design-PubMed and Embase were screened for new references using the original search strings. Studies were required to be population-based, to include $\geq 200$ individuals, to have response rates $250 \%$ and recall periods $<12$ months. GERD was defined as heartburn and/or regurgitation on at least 1 day a week, or according to the Montreal definition, or diagnosed by a clinician. Temporal and geographic trends in disease prevalence were examined using a Poisson regression model.
\end{abstract}

Results-16 studies of GERD epidemiology published since the original review were found to be suitable for inclusion (15 reporting prevalence and one reporting incidence), and were added to the 13 prevalence and two incidence studies found previously. The range of GERD prevalence estimates was $18.1 \%-27.8 \%$ in North America, $8.8 \%-25.9 \%$ in Europe, $2.5 \%-7.8 \%$ in East Asia, 8.7\%-33.1\% in the Middle East, $11.6 \%$ in Australia and 23.0\% in South America. Incidence per 1000 person-years was approximately 5 in the overall UK and US populations, and 0.84 in

\footnotetext{
Copyright Article author (or their employer) 2013.

Correspondence to Dr Hashem B El-Serag, Section of Gastroenterology and Hepatology, Houston VA Medical Center, and Baylor College of Medicine, 2002 Holcombe Boulevard (152), Houston, TX 77030, USA; hasheme@ bcm.edu.

To cite: El-Serag HB, Sweet S, Winchester CC, et al. Gut Published Online First: [please include Day Month Year] doi:10.1136/ gutjnl-2012-304269

Contributors All authors contributed to the development of the search strategy. Database searches were performed and screened by SS. Statistical analyses were conducted by HBE-S. All authors were involved in the interpretation of the data, drafting and review of the manuscript, and approval of the final version.

Competing interests Hashem B El-Serag and John Dent have undertaken consultancy work for AstraZeneca, and John Dent contributes to postgraduate activities sponsored by AstraZeneca. Stephen Sweet and Christopher C Winchester are employees of Oxford PharmaGenesis, which has received funding from AstraZeneca.

Provenance and peer review Not commissioned; externally peer reviewed.

Data sharing statement Information regarding this systematic review is available from the corresponding author, as detailed in the manuscript.
} 
paediatric patients aged 1- 17 years in the UK. Evidence suggests an increase in GERD prevalence since 1995 ( $\mathrm{p}<0.0001$ ), particularly in North America and East Asia.

Conclusions-GERD is prevalent worldwide, and disease burden may be increasing. Prevalence estimates show considerable geographic variation, but only East Asia shows estimates consistently lower than $10 \%$.

\section{INTRODUCTION}

In 2005, two of the present authors contributed to a systematic review of population-based epidemio-logical studies of gastro-oesophageal reflux disease (GERD). ${ }^{1}$ In total, 15 studies were identified that together reported an approximate prevalence of GERD of 10\%-20\% in Europe and the USA, and of less than 5\% in Asia. GERD incidence was reported to be approximately 5 per 1000 person-years in both the UK and the USA. Factors reported to be associated with GERD included comorbid respiratory disease, chest pain and obesity, and a family history of the disease. At the time, it was noted that there was a lack of consensus as to the definition of GERD and, in order to overcome this, included papers were limited to those that defined GERD as either the presence of symptoms of heart-burn or regurgitation on at least 1 day a week if assessed by a questionnaire, or diagnosed by a physician.

There were, however, a number of limitations to the findings of the original review. The majority of the included studies ( 12 of 15) were carried out in Europe or the USA, with only three being from Asia (Hong Kong or China) and none from other parts of the world. This is a similar pattern to that seen in a systematic review specifically reporting geographical and racial differences in GERD epidemiology. ${ }^{2}$ There was also very limited information on the prevalence of GERD in children, with only one prevalence study including individuals under the age of 17 years. Data on the incidence of GERD were also rare. Since 2005, many epidemio-logical studies of GERD have been published and, in 2007, another systematic review suggested that the prevalence of GERD is increasing. ${ }^{3}$ In addition, the Montreal definition of the disease was published in 2006, which defines GERD as 'troublesome symptoms and/or complications' resulting from gastro-oesophageal reflux. ${ }^{4}$ This consensus also states that, in epidemiological studies, GERD may be defined as mild symptoms occurring on two or more days per week, or moderate-to-severe symptoms occurring on one or more days per week (a threshold often considered troublesome by patients).

In order to take account of more recently published studies and the consensus definition of the disease, we have undertaken an update of the original review. The search strategy, inclusion and exclusion criteria were kept as similar as possible to those used previously. Here, we report the results of this updated systematic literature review.

\section{METHODS}

\section{Study selection}

Searches were performed in PubMed and Embase for studies published between 1 July 2004 and 20 February 2011 which included the terms 'gastroesophageal reflux', 'GERD', 'GORD', 'heartburn', 'esophagitis' or 'oesophagitis' combined with 'epidemiology', 'epidemiological', 'prevalence', 'incidence' or 'population' in the title, abstract or list of 
medical subject heading terms (figure 1). Studies were limited to those published in English, and reviews, meta-analyses and editorials were excluded. As, historically, the quality of epidemiological studies of GERD has varied widely, selection criteria for the original review were chosen based on their face validity, with the aim of identifying the largest possible number of comparable, high quality studies. These criteria were also applied to the current review. To be suitable for inclusion, studies had to be population-based, and GERD diagnosis had to be by a clinician or by a symptom-based questionnaire with a recall period of no more than 12 months. Study populations were required to include at least 200 individuals and, to minimise response bias, response rates were required to be greater than $50 \%$. As the prevalence of GERD decreases as the frequency and severity threshold for reporting symptoms increases, ${ }^{56}$ GERD was defined as heartburn and/or regurgitation of any severity on at least 1 day a week (found review to be the most commonly used definition of GERD), or diagnosed by a clinician.

In addition, we also included studies that defined GERD according to the Montreal definition. Studies of paediatric patient populations were required to exclude infants aged $<1$ year or to report the results for infants separately from the rest of the population. All studies identified in the 2005 review were also included. This systematic review was carried out in accordance with the Preferred Reporting Items for Systematic Reviews and Meta-Analyses guidelines. $^{7}$

\section{Statistical analyses}

Poisson regression models were used to analyse the effect of study year (or, when this information was not available, the year of study publication) and the continent on which studies were conducted on the reported prevalence of GERD. Study year was analysed as a categorical variable, with categories: pre-1995, 1995-1999, 2000-2004 and 2005-2009, and the earliest category was used when a study was carried out over a period of time encompassing two categories. When an estimate of the prevalence of at least weekly heartburn and/or regurgitation was not available, the prevalence of at least weekly heartburn was used as a measure of the prevalence of GERD. In two separate sensitivity analyses, we used the prevalence of regurgitation as a measure of the prevalence of GERD when the prevalence of heartburn and/or regurgitation was not available, or excluded all studies in which the prevalence of at least weekly heartburn and/or regurgitation was not reported. Estimates of relative risk were calculated as prevalence rate ratios (RRs) with $95 \%$ CIs. p Values were calculated using the Wald $\chi^{2}$ test. We also evaluated temporal trends in disease prevalence separately in studies conducted in North America, Europe and East Asia. When assessing temporal trends in GERD prevalence worldwide, the Poisson regression model was adjusted for the continent on which a study was conducted.

\section{RESULTS}

\section{Search results}

After the removal of duplicate papers identified in both PubMed and Embase, 2183 articles were found in the database searches for papers published since July 2004. These were screened for relevance based on titles and abstracts, after which 85 full references were 
obtained for further analysis. After review, 16 were found to be suitable for inclusion (figure 1). Studies were most commonly excluded for not being population-based (31 studies) or not reporting the prevalence of GERD defined as at least weekly heartburn and/or regurgitation, conforming to the Montreal definition, or diagnosed by a clinician (24 studies). Fifteen of the included studies assessed GERD prevalence, while one reported GERD incidence (tables 1 and 3).

\section{Prevalence of GERD}

North America-Only one of the 15 studies published since the original review reported GERD prevalence in the USA. This was a survey carried out in 2003 among residents aged 20-95 years in Olmsted County, Minnesota, USA (table 1). ${ }^{8}$ A bowel-disease questionnaire that included questions assessing reflux symptoms was sent to 4194 individuals, and 2273 questionnaires (54.2\%) were completed and returned. A total of 411 individuals (18.1\%) had GERD, defined as at least weekly heartburn and/or regurgitation. This prevalence estimate is similar to that reported in papers published in 1997 and 1999 assessing the same underlying population (approximately $20 \%$ in both studies), ${ }^{910}$ and most likely greater than that reported in another Olmsted County paper from 1992 that assessed the prevalence of at least weekly heartburn (13.2\%) and at least weekly regurgitation (6.5\%) separately, but did not report the prevalence of at least weekly heartburn and/or regurgitation together. ${ }^{11}$ The population of Olmsted County is predominantly Caucasian (approximately 90\%, compared with approximately $80 \%$ overall in the USA ${ }^{12}$ ), and is sociodemographically similar to the US white population. Another paper included in the 2005 review reported the prevalence of at least weekly heart-burn and/or regurgitation in an employed population in the southern USA to be $27.8 \% .^{13}$ Taken together, the four studies that reported the prevalence of GERD defined as at least weekly heartburn and/or regurgitation (not heartburn and regurgitation separately) in the original review and the updated searches show the prevalence of GERD in the USA to be $18.1 \%-27.8 \%$ (sample size-weighted mean $19.8 \%$ ).

\section{South America}

Since July 2004 one study carried out in South America has been published that meets the inclusion criteria for our review. Chiocca et al ${ }^{14}$ studied the prevalence of GERD in 1998 (published in 2005) among 839 individuals (response rate 83.9\%) living in 17 representative geographical regions of Argentina with different population densities. The population for this study was selected from working and elderly populations in companies and community centres, and therefore did not include unemployed individuals of working age. The prevalence of at least weekly heartburn and/or regurgitation was $23.0 \%$, while the prevalence of at least weekly heartburn was $16.9 \%$ and the prevalence of at least weekly regurgitation was $16.5 \%$. No South American studies were included in the original review.

\section{Europe}

Two studies of the prevalence of GERD in Europe have been published since the original review. Lofdahl et al ${ }^{15}$ carried out a postal questionnaire survey in a random sample selected from the general Swedish population (in a narrow age-range of 40-59 years), and reported a prevalence of at least weekly heart-burn and/or regurgitation of $8.8 \%$. A total of 
1483 individuals participated in this study, which represented a response rate of $62.5 \%$ to an original invited population of 2373. Sampling was based on the age and sex distribution of oesophageal adenocarcinoma according to the Swedish Cancer Registry. The second paper reported the results of the Kalixanda study, also carried out in Sweden, in the Kalix and Haparanda communities in the North of the country. ${ }^{16}$ A total of 1000 of 1365 eligible individuals approached (response rate 73.3\%) answered a postal questionnaire assessing reflux symptoms. Weekly heartburn and/or regurgitation were experienced by 259 of participants (25.9\%).

The original review included six European studies ${ }^{17-22}$ which reported a prevalence of GERD ranging from $9.8 \%$ to $18 \%$. Including the results in our updated review, therefore, the range of prevalence estimates has broadened slightly to $8.8 \%-25.9 \%$ (sample size-weighted mean $15.2 \%$ ), with a tendency for GERD to be more prevalent in northern than southern Europe.

Middle East-Seven studies have been published since the original review reporting the prevalence of GERD in the Middle East, including five in Iran, ${ }^{23-27}$ one in Turkey ${ }^{28}$ and one in Israel. ${ }^{29}$ Of the five studies based in Iran, four were carried out in Tehran ${ }^{24-27}$ and one among the Qashqai migrating nomads of Fars. ${ }^{23}$ In the four studies based in Tehran, the prevalence of at least weekly heart-burn and/or regurgitation varied between $8.7 \%$ and $21.2 \%$, with two studies reporting a prevalence of approximately $20 \%$, and the other two reporting a prevalence of approximately $10 \%$. The reason for the differences in the results of these studies is unclear, but may be related to differences in the definitions of heartburn and regurgitation used. Two of the four studies conducted in Tehran also reported the prevalence of at least weekly heartburn $(2.2 \%-9.6 \%)$ and regurgitation $(7.2 \%-12.2 \%)$ separately. ${ }^{2426}$

The survey of 717 (response rate 95.9\%) migrating nomads in Fars province in the south of the country found the prevalence of at least weekly heartburn and/or regurgitation to be $33.1 \%{ }^{23}$ This estimate is higher than that reported in the other worldwide studies of GERD prevalence, and it should be noted that, as migrating nomads represent a distinct racial subgroup, these results are unlikely to be generalisable to the general Iranian population. The Qashqai migrating nomads move between winter quarters near the Persian Gulf and summer quarters in the Zagros Mountains in the north of Fars province. They live in tents, are highly physically active and have a different diet from urban dwellers. The survey, which had a 12-month recall period, was conducted using face-to-face interviews during May to October 2006 while the population was living in its summer quarters.

In Turkey, Kitapcioglu et $a l^{28}$ reported the results of a survey carried out in 1998-1999 (published in 2007) of 630 individuals (response rate 83.1\%) aged over 20 years in the Asian town of Menderes. The prevalence of at least weekly heartburn and/or regurgitation was $20 \%$, while the prevalence of at least weekly heartburn was $10 \%$, and the prevalence of at least weekly regurgitation was $15.6 \%$. In Israel, Sperber et $a l^{29}$ reported the prevalence of GERD in a Jewish population (981 individuals, response rate 80.3\%). The prevalence of at least weekly heartburn and/or regurgitation was $9.3 \%$. It should be noted that as this study did not include any individuals who were not Jewish, it is not generalisable to the population of Israel as a whole (approximately $75 \%$ Jewish $^{12}$ ). 
Estimates of the prevalence of GERD in the Middle East therefore ranged from $8.7 \%$ to $33.1 \%$ (sample size-weighted mean $14.4 \%$ ) or $8.7 \%-21.2 \%$ if the migrating nomads of Fars in Iran are excluded (sample size-weighted mean 13.4\%). No studies reporting the prevalence of GERD in the Middle East were available at the time of the original review.

East Asia-In total, three East Asian studies of the prevalence of GERD have been published since the original review: two in China ${ }^{3031}$ and one in South Korea. ${ }^{32}$ Of the studies in China, Chen et $\mathrm{l}^{30}$ reported the results of a 2003 survey of 3338 people (response rate $95.0 \%$ ) aged 18-90 years and randomly selected from the cities of Guangzhou and Huizhou in southern China. The prevalence of at least weekly heartburn was $2.2 \%$, the prevalence of at least weekly regurgitation was $7.0 \%$, and the prevalence of at least weekly heartburn and/or regurgitation was $7.8 \%$. Also in China, the Systematic Investigation of Gastrointestinal Diseases in China (SILC) study reported from a survey carried out in 2007 of 16078 individuals (response rate 89.3\%) aged 18-80 years randomly sampled from urban and rural areas of Shanghai, Beijing, Wuhan, Xi' an and Guangzhou. ${ }^{31}$ Overall, the prevalence of at least weekly heart-burn and/or regurgitation was $5.2 \%$, varying between the different regions from $3.2 \%$ to $7.5 \%$. In this study, the prevalence of at least weekly heartburn in the overall population was $1.8 \%$, while the prevalence of at least weekly regurgitation was $4.2 \%$. This paper also reported the prevalence of GERD defined according to the Montreal definition for use in epidemiological studies. ${ }^{4}$ The overall prevalence of Montreal-defined GERD was 3.1\%, varying between $1.7 \%$ and $5.1 \%$ in the different regions. This was the only study included in this review to use the Montreal criteria for epidemiological studies to define GERD. ${ }^{4}$

In South Korea, Cho et al $^{32}$ reported from a survey carried out between 2000 and 2001 of 1417 individuals (response rate 78.4\%) aged 18-69 years from Asan-si in Chungcheongnam-do Province. The prevalence of at least weekly heartburn and/or regurgitation was $3.5 \%$, the prevalence of at least weekly heart-burn was $2.0 \%$ and the prevalence of at least weekly regurgitation was $2.0 \%$. The prevalence of GERD reported in East Asian studies published since July 2004 therefore ranged from 3.5\% to 7.8\%, comparable with the prevalence range of 2.5\%-4.8\% reported in studies in East Asian populations included in the original review. ${ }^{33-35}$ Across both reviews, the range of prevalence estimates was $2.5 \%-7.8 \%$ (sample size-weighted mean $5.2 \%$ ).

Australasia-One study has been published since the original review that reported the prevalence of GERD in Australia. ${ }^{36}$ This study assessed the prevalence of GERD among 672 individuals (response rate $72.6 \%$ ) living in western Sydney, using a validated postal questionnaire with a recall period of 12 months. The prevalence of at least weekly heartburn and/or regurgitation was $11.6 \%$.

\section{Trends in the prevalence of GERD}

We analysed the effect of the year a study was conducted (or, when this information was not available, the year of publication) on the estimate of GERD prevalence using a Poisson regression model adjusted for the continent on which the study was conducted. When a study did not include an estimate of the prevalence of at least weekly heartburn and/or 
regurgitation, the prevalence of at least weekly heartburn was used as a measure of the prevalence of GERD instead. There was evidence for a statistically significant increase in the prevalence of GERD worldwide when studies conducted before 1995 were compared with those published after 1995. However, when comparing studies published in 1995-1999, 2000-2004 and 2005-2009, this increase appears to level off, with very similar RRs for all of these periods when prevalence estimates are compared with those from studies published before 1995 (table 2). It should also be noted that only three studies were carried out before $1995,{ }^{112021}$ and only one of these included an estimate of the prevalence of at least weekly heartburn and/or regurgitation. ${ }^{20}$

To avoid bias in the results due to the use of the prevalence of at least weekly heartburn when no estimate of the prevalence of at least weekly heartburn and/or regurgitation was available, two sensitivity analyses were conducted. In the first, the prevalence of at least weekly regurgitation was used instead of at least weekly heartburn in such studies. In the second, all studies that did not include an estimate of the prevalence of at least weekly heartburn and/or regurgitation were excluded from the analysis. In both cases, statistically significant increases in GERD prevalence of a similar magnitude were observed as in the original analysis (data not shown).

We also examined temporal trends in GERD prevalence among the five studies from North America, and found that the prevalence of GERD was significantly higher in studies carried out after 1995 than in those carried out before 1995 (RR 1.50; 95\% CI 1.23 to 1.84 for studies conducted between 1995 and 1999 vs studies conducted before 1995, and RR 1.50; 95\% CI 1.22 to 1.84 for studies conducted between 2000 and 2004 vs studies conducted before 1995). Similarly, for the six studies conducted in East Asia, the prevalence of GERD was significantly higher in studies conducted between 2000 and 2004 (RR 1.49, 95\% CI 1.26 to 1.76) and between 2005 and 2009 (RR 1.47, 95\% CI 1.27 to 1.69) than in those conducted between 1995 and 1999. For the eight studies conducted in Europe, there was a significant increase in prevalence estimates in studies conducted during 2005-2009 compared with those conducted before 1995 (RR 1.52; 95\% CI 1.06 to 2.19), but no significant increase when studies conducted in 1995-1999 or 2000-2004 were compared with those conducted before 1995 .

In a further analysis in which the prevalence of GERD was compared between continents, prevalence was shown to be the highest in North America, followed by the Middle East, Europe and East Asia (table 2). As only single studies were available from South America and Australasia, these continents were not included in the analysis. When the two sensitivity analyses were conducted as described above, similar geographic trends in the prevalence of GERD were observed (data not shown).

\section{Incidence of GERD}

Only one study of GERD incidence published since the original review was identified, which reported incidence in paediatric patients aged 1-17 years in the UK from 2000 to $2005^{37}$ (table 3). This study by Ruigómez et al used The Health Improvement Network, a large primary care database containing anonymised information on approximately 2.3 million patients currently registered with participating primary care practitioners (PCPs) 
(approximately $4 \%$ of the total UK population). Included patients were required to be aged 1-17 years, to have been registered with their PCP for at least 1 year before the start of the study period, and to have no diagnosis of GERD prior to a first diagnosis in the period 2000-2005. The overall incidence was 0.84 per 1000 person-years. In comparison, two studies of GERD incidence were included in the 2005 review, one from the UK and one from the USA. ${ }^{3839}$ Both reported disease incidence in either adult patients or the adult and paediatric populations considered together, and found the incidence of GERD to be approximately 5 per 1000 person-years. The incidence of GERD in children over 1 year of age may therefore be considerably lower than that in the adult population. It should be noted that the study of Kotzan et al from the USA used data from a Medicaid claims database, and is therefore only representative of the low-income population who receive Medicaid.

\section{Factors associated with GERD}

While it was not the primary goal of this systematic review to analyse GERD risk factors, several of the studies published in the original review and since July 2004 reported the results of multivariate analyses carried out to identify factors independently associated with GERD (see online supplementary table S1). Factors reported in different studies are variable and results are not universally consistent. However, three studies offered evidence for an association between having GERD symptoms and having a genetically related family member with gastrointestinal symptoms, ${ }^{101931}$ while one study showed no association between having GERD and having a spouse with a history of gastrointestinal symptoms. ${ }^{10}$ One study failed to show an association between having GERD and having an immediate family history of the disease.$^{24}$ Only one study showed that age is associated with GERD, and no studies showed that male sex is associated with GERD. Evidence for associations of GERD with smoking and obesity was conflicting. A complete list of factors assessed and associations found in each study is presented in online supplementary table S1.

\section{DISCUSSION}

In this update to the 2005 review of GERD epidemiology, we have identified 15 populationbased studies published since the original literature searches that assessed the prevalence of GERD, and one study that assessed GERD incidence. In total, our review now comprises data from 31 studies, including 28 studies of GERD prevalence that together involved more than 65000 individuals and three studies of GERD incidence that involved approximately 5.5 million individuals. The geographic profile of studies of GERD prevalence and incidence has changed considerably since 2005. While in the original review 12 of 15 included studies were carried out in Europe or the USA, with the remaining three being based in East Asia, only one of the new studies reported here was based in the USA, two in Europe and three in East Asia. Most new studies have been carried out in regions not previously assessed, particularly the Middle East.

Figure 2 shows a map indicating sample-size weighted mean estimates of the prevalence of at least weekly heartburn and/or regurgitation in each country where such an estimate was available. The range of GERD prevalence estimates in Europe and the USA has broadened slightly from those reported in the original review, with estimates including both new and 
older studies ranging from $18.1 \%$ to $27.8 \%$ in the USA and $8.8 \%$ to $25.9 \%$ in Europe. It should be noted that of the studies conducted in the USA, El Serag et al assessed GERD prevalence only in employed individuals, and reported the highest disease prevalence of $27.8 \% .{ }^{13}$ All other studies from the USA reported prevalence estimates of approximately $20 \%$, but were conducted in Olmsted County, the population of which has little representation from the USA's black, Latin-American or Asian-American populations. The sample size weighted mean estimate of GERD prevalence in the USA was $19.8 \%$.

Estimates of GERD prevalence in the newly studied regions of the Middle East, Australasia and South America are also broad, but appear to be comparable with the 10\%-30\% range found in Europe and the USA. The highest prevalence estimate, of 33.1\%, was observed in the Qashqai migrating nomads in the Fars province of Iran, a population which may not be representative of the whole country. Excluding this study, prevalence estimates in the Middle East are approximately 10\%-20\%. Prevalence in East Asia is still substantially lower than that in Western populations $(2.5 \%-7.8 \%)$. In statistical tests, including sensitivity analyses conducted to avoid bias in the results, we saw evidence that the overall prevalence of GERD has increased since 1995. Our findings are in line with a previous systematic review that also reported an increase in GERD prevalence with time, ${ }^{3}$ and with the results of a large Norwegian longitudinal population-based study of the prevalence of gastrooesophageal reflux symptoms that unfortunately did not meet the criteria for inclusion in this review, because the symptom assessment criteria could not be matched with the included studies (the Nord-Trondelag Health Study (HUNT)). ${ }^{4041}$

We have also assessed the results of multivariate analyses carried out in the included studies to identify factors associated with the presence of GERD, and have found good evidence for familial clustering of GERD. Conversely, we found little evidence that age is associated with GERD, in agreement with the findings of a recent systematic review, ${ }^{42}$ and no evidence that male sex is associated with GERD (two studies showed that female sex was positively associated with GERD). Evidence regarding associations of GERD with other previously studied factors including smoking and obesity were variable. However, it should be noted that our review was not primarily designed to examine GERD risk factors, and excluded studies that did not report population-based estimates of disease incidence or prevalence. Potentially important variables such as Helicobacter pylori infection, ${ }^{43}$ abdominal obesity and hiatus hernia ${ }^{44}$ are dif-ficult to survey using the questionnaire-based methods commonly used in the studies included in this review.

A strength of this review was the stringent selection criteria applied to the included studies, with a particular focus on the consistency of disease definition, to ensure that GERD prevalence estimates are comparable. The definition of GERD as at least weekly symptoms was found while carrying out the original review to be the measure most often used in GERD epidemiological studies, and therefore allowed for the inclusion of as many studies as possible. ${ }^{1}$ It is also approximately in line with the Montreal definition of GERD, which states that 'troublesome symptoms' may be considered to be moderate-to-severe symptoms occurring on one or more days per week (although our definition also includes mild symptoms occurring on one or more days per week). ${ }^{4}$ Our estimates will, however, include some subjects who have heartburn generated by mechanisms other than reflux, and will not 
include those with reflux oesophagitis whose symptoms do not meet the threshold for GERD diagnosis.

Some population-based studies were excluded on the basis that they did not define GERD as at least weekly heartburn and/ or regurgitation, although they were otherwise of high quality. 40

In addition, in order to include as many studies as possible that met the disease definition selection criterion, we used a relatively generous definition of 'population-based', including any studies where patients were not selected on the basis of their health status or healthcare consultation or referral. Studies of nationally representative populations have been conducted only in Europe, and data from local populations in other countries (such as Olmsted County in the USA) may not be generalisable to the country as a whole. It is notable that the two highest prevalence estimates found in the USA and the Middle East both tested populations not fully representative of the general populations of the countries studied. ${ }^{1323}$ Excluding these two studies, prevalence estimates for all geographic areas except East Asia are in the approximate 10\%-20\% range, with the exception of one study carried out in Europe. ${ }^{16}$

Although we have included a good number of studies from North America, Europe, the Middle East and Asia, studies meeting our inclusion criteria are still lacking from important developed countries such as Japan, and emerging economies such as Brazil, Russia, India and Africa. There are also still few data regarding the prevalence of GERD in paediatric populations, and few studies of GERD incidence. In addition, although we have included studies of disease incidence, we have not been able to address disease disappearance. However, a high chronicity of the disease has been previously reported, ${ }^{45}$ which is consistent with the low rate of symptom disappearance seen in studies not eligible for inclusion in this review. ${ }^{4146}$ Disease disappearance is likely to be counterbalanced by disease incidence in creating a constant or increasing overall disease prevalence.

In summary, the results of this updated systematic review show that GERD remains a prevalent disease worldwide, with only East Asia showing prevalence estimates consistently below $10 \%$. The high prevalence of the disease has potentially serious societal consequences, since the pain and discomfort caused by GERD adversely impacts many aspects of patients' lives (including their productivity at work).$^{47}$ GERD is also a risk factor for the development of Barrett's oesophagus and oesophageal adenocarcinoma, ${ }^{48}$ conditions that are currently rare in Asia, but are increasing in Western populations. ${ }^{49}$

\section{Acknowledgments}

Funding This work was supported in part by Texas Digestive Disease Center grants NIH DK58338 and NIDDK K24-04-107 to Dr El-Serag and by the Houston VA Health Services R\&D Center of Excellence (HFP90-020). The views expressed in this article are those of the author(s) and do not necessarily represent the views of the Department of Veterans Affairs. Stephen Sweet and Christopher C Winchester are employees of Oxford PharmaGenesis, which received funding from AstraZeneca R\&D, Mölndal, Sweden for its role in the preparation of this systematic review. 


\section{REFERENCES}

1. Dent J, El-Serag HB, Wallander MA, et al. Epidemiology of gastro-oesophageal reflux disease: a systematic review. Gut. 2005; 54:710-17. [PubMed: 15831922]

2. Sharma P, Wani S, Romero Y, et al. Racial and geographic issues in gastroesophageal reflux disease. Am J Gastroenterol. 2008; 103:2669-80. [PubMed: 19032462]

3. El-Serag HB. Time trends of gastroesophageal reflux disease: a systematic review. Clin Gastroenterol Hepatol. 2007; 5:17-26. [PubMed: 17142109]

4. Vakil N, Veldhuyzen van Zanten S, Kahrilas P, et al. The Montreal definition and classification of gastro-esophageal reflux disease (GERD) - a global evidence-based consensus. Am J Gastroenterol. 2006; 101:1900-20. [PubMed: 16928254]

5. Ronkainen J, Aro P, Storskrubb T, et al. Gastro-oesophageal reflux symptoms and health-related quality of life in the adult general population — the Kalixanda study. Aliment Pharmacol Ther. 2006; 23:1725-33. [PubMed: 16817916]

6. Wiklund I, Carlsson J, Vakil N. Gastroesophageal reflux symptoms and well-being in a random sample of the general population of a Swedish community. Am J Gastroenterol. 2006; 101:18-28. [PubMed: 16405529]

7. Liberati A, Altman DG, Tetzlaff J, et al. The PRISMA statement for reporting systematic reviews and meta-analyses of studies that evaluate healthcare interventions: explanation and elaboration. BMJ. 2009; 339:b2700. [PubMed: 19622552]

8. Jung HK, Halder S, McNally M, et al. Overlap of gastro-oesophageal reflux disease and irritable bowel syndrome: prevalence and risk factors in the general population. Aliment Pharmacol Ther. 2007; 26:453-61. [PubMed: 17635380]

9. Locke GR III, Talley NJ, Fett SL, et al. Prevalence and clinical spectrum of gastroesophageal reflux: a population-based study in Olmsted County, Minnesota. Gastroenterology. 1997; 112:1448-56. [PubMed: 9136821]

10. Locke GR III, Talley NJ, Fett SL, et al. Risk factors associated with symptoms of gastroesophageal reflux. Am J Med. 1999; 106:642-9. [PubMed: 10378622]

11. Talley NJ, Zinsmeister AR, Schleck CD, et al. Dyspepsia and dyspepsia subgroups: a populationbased study. Gastroenterology. 1992; 102:1259-68. [PubMed: 1551533]

12. The World Factbook. [18 Feb 2013] Central Intelligence Agency. 2012. https://www.cia.gov/ library/publications/the-world-factbook/index.html

13. El-Serag HB, Petersen NJ, Carter J, et al. Gastroesophageal reflux among different racial groups in the United States. Gastroenterology. 2004; 126:1692-9. [PubMed: 15188164]

14. Chiocca JC, Olmos JA, Salis GB, et al. Prevalence, clinical spectrum and atypical symptoms of gastro-oesophageal reflux in Argentina: a nationwide population-based study. Aliment Pharmacol Ther. 2005; 22:331-42. [PubMed: 16098000]

15. Lofdahl HE, Lane A, Lu Y, et al. Increased population prevalence of reflux and obesity in the United Kingdom compared with Sweden: a potential explanation for the difference in incidence of esophageal adenocarcinoma. Eur J Gastroenterol Hepatol. 2011; 23:128-32. [PubMed: 21178778]

16. Ronkainen J, Aro P, Storskrubb T, et al. High prevalence of gastroesophageal reflux symptoms and esophagitis with or without symptoms in the general adult Swedish population: a Kalixanda study report. Scand J Gastroenterol. 2005; 40:275-85. [PubMed: 15932168]

17. Diaz-Rubio M, Moreno-Elola-Olaso C, Rey E, et al. Symptoms of gastro-oesophageal reflux: prevalence, severity, duration and associated factors in a Spanish population. Aliment Pharmacol Ther. 2004; 19:95-105. [PubMed: 14687171]

18. Isolauri J, Laippala P. Prevalence of symptoms suggestive of gastro-oesophageal reflux disease in an adult population. Ann Med. 1995; 27:67-70. [PubMed: 7742002]

19. Mohammed I, Cherkas LF, Riley SA, et al. Genetic influences in gastro-oesophageal reflux disease: a twin study. Gut. 2003; 52:1085-9. [PubMed: 12865263]

20. Terry P, Lagergren J, Wolk A, et al. Reflux-inducing dietary factors and risk of adenocarcinoma of the esophagus and gastric cardia. Nutr Cancer. 2000; 38:186-91. [PubMed: 11525596] 
21. Thompson WG, Heaton KW. Heartburn and globus in apparently healthy people. Can Med Assoc J. 1982; 126:46-8. [PubMed: 7059872]

22. Valle C, Broglia F, Pistorio A, et al. Prevalence and impact of symptoms suggestive of gastroesophageal reflux disease. Dig Dis Sci. 1999; 44:1848-52. [PubMed: 10505724]

23. Mostaghni A, Mehrabani D, Khademolhosseini F, et al. Prevalence and risk factors of gastroesophageal reflux disease in Qashqai migrating nomads, southern Iran. World J Gastroenterol. 2009; 15:961-5. [PubMed: 19248195]

24. Nasseri-Moghaddam S, Mofid A, Ghotbi MH, et al. Epidemiological study of gastro-oesophageal reflux disease: reflux in spouse as a risk factor. Aliment Pharmacol Ther. 2008; 28:144-53. [PubMed: 18410559]

25. Nouraie M, Radmard AR, Zaer-Rezaii H, et al. Hygiene could affect GERD prevalence independently: a population-based study in Tehran. Am J Gastroenterol. 2007; 102:1353-60. [PubMed: 17437507]

26. Nouraie M, Razjouyan H, Assady M, et al. Epidemiology of gastroesophageal reflux symptoms in Tehran, Iran: a population-based telephone survey. Arch Iran Med. 2007; 10:289-94. [PubMed: 17604463]

27. Solhpour A, Pourhoseingholi MA, Soltani F, et al. Gastro-esophageal reflux symptoms and body mass index: no relation among the Iranian population. Indian J Gastroenterol. 2008; 27:153-5. [PubMed: 18974465]

28. Kitapcioglu G, Mandiracioglu A, Bor CC, et al. Overlap of symptoms of dyspepsia and gastroesophageal reflux in the community. Turk J Gastroenterol. 2007; 18:14-9. [PubMed: 17450489]

29. Sperber AD, Halpern Z, Shvartzman P, et al. Prevalence of GERD symptoms in a representative Israeli adult population. J Clin Gastroenterol. 2007; 41:457-61. [PubMed: 17450026]

30. Chen M, Xiong L, Chen H, et al. Prevalence, risk factors and impact of gastroesophageal reflux disease symptoms: a population-based study in South China. Scand J Gastroenterol. 2005; 40:75967. [PubMed: 16118911]

31. He J, Ma X, Zhao Y, et al. A population-based survey of the epidemiology of symptom-defined gastroesophageal reflux disease: the Systematic Investigation of Gastrointestinal Diseases in China. BMC Gastroenterol. 2010; 10:94. [PubMed: 20707933]

32. Cho YS, Choi MG, Jeong JJ, et al. Prevalence and clinical spectrum of gastroesophageal reflux: a population-based study in Asan-si, Korea. Am J Gastroenterol. 2005; 100:747-53. [PubMed: 15784014]

33. Hu WH, Wong WM, Lam CL, et al. Anxiety but not depression determines health care-seeking behaviour in Chinese patients with dyspepsia and irritable bowel syndrome: a population-based study. Aliment Pharmacol Ther. 2002; 16:2081-8. [PubMed: 12452941]

34. Pan G, Xu G, Ke M, et al. Epidemiological study of symptomatic gastroesophageal reflux disease in China: Beijing and Shanghai. Chin J Dig Dis. 2000; 1:2-8.

35. Wong WM, Lai KC, Lam KF, et al. Prevalence, clinical spectrum and health care utilization of gastro-oesophageal reflux disease in a Chinese population: a population-based study. Aliment Pharmacol Ther. 2003; 18:595-604. [PubMed: 12969086]

36. Eslick GD, Talley NJ. Gastroesophageal reflux disease (GERD): risk factors, and impact on quality of life-a population-based study. J Clin Gastroenterol. 2009; 43:111-17. [PubMed: 18838922]

37. Ruigómez A, Wallander MA, Lundborg P, et al. Gastroesophageal reflux disease in children and adolescents in primary care. Scand J Gastroenterol. 2010; 45:139-46. [PubMed: 19961345]

38. Kotzan J, Wade W, Yu HH. Assessing NSAID prescription use as a predisposing factor for gastroesophageal reflux disease in a Medicaid population. Pharm Res. 2001; 18:1367-72. [PubMed: 11683254]

39. Ruigómez A, García Rodríguez LA, Wallander MA, et al. Natural history of gastro-oesophageal reflux disease diagnosed in general practice. Aliment Pharmacol Ther. 2004; 20:751-60. [PubMed: 15379835]

40. Nilsson M, Johnsen R, Ye W, et al. Prevalence of gastro-oesophageal reflux symptoms and the influence of age and sex. Scand J Gastroenterol. 2004; 39:1040-5. [PubMed: 15545159] 
41. Ness-Jensen E, Lindam A, Lagergren J, et al. Changes in prevalence, incidence and spontaneous loss of gastro-oesophageal reflux symptoms: a prospective population-based cohort study, the HUNT study. Gut. 2012; 61:1390-7. [PubMed: 22190483]

42. Becher A, Dent J. Systematic review: ageing and gastro-oesophageal reflux disease symptoms, oesophageal function and reflux oesophagitis. Aliment Pharmacol Ther. 2011; 33:442-54. [PubMed: 21138458]

43. Raghunath A, Hungin AP, Wooff D, et al. Prevalence of Helicobacter pylori in patients with gastro-oesophageal reflux disease: systematic review. BMJ. 2003; 326:737. [PubMed: 12676842]

44. Dent J, Becher A, Sung J, et al. Systematic review: patterns of reflux-induced symptoms and esophageal endoscopic findings in large-scale surveys. Clin Gastroenterol Hepatol. 2012; 10:863873. [PubMed: 22401904]

45. Armstrong D. Gastroesophageal reflux disease-a chronic, persistent disease: a systematic review of the literature [abstract]. Gastroenterology. 2008; 134(4 Suppl 1):S1092.

46. Talley NJ, Weaver AL, Zinsmeister AR, et al. Onset and disappearance of gastrointestinal symptoms and functional gastrointestinal disorders. Am J Epidemiol. 1992; 136:165-77. [PubMed: 1415139]

47. Liker H, Hungin AP, Wiklund I. Management of reflux disease in primary care: the patient perspective. J Am Board Fam Pract. 2005; 18:393-400. [PubMed: 16148249]

48. Dent J. Barrett's esophagus: a historical perspective, an update on core practicalities and predictions on future evolutions of management. J Gastroenterol Hepatol. 2011; 26:11-30. [PubMed: 21199510]

49. Chang CY, Cook MB, Lee YC, et al. Current status of Barrett's esophagus research in Asia. J Gastroenterol Hepatol. 2011; 26:240-6. [PubMed: 21155883] 


\section{Significance of this study}

\section{What is already known on this subject?}

In 2005, a systematic review of population-based studies (involving two of the present authors) found the prevalence of gastro-oesophageal reflux disease (GERD) to be $10 \%-20 \%$ in Europe and the USA, and less than 5\% in East Asia.

- The incidence of the disease was approximately 5 per 1000 person-years.

- We have updated the original review incorporating data from studies published between July 2004 and February 2011.

\section{What are the new findings?}

- The range of studied geographical areas has expanded considerably and now includes the Middle East, Australia and South America.

- GERD prevalence estimates vary widely, but only East Asian studies show prevalence estimates consistently less than $10 \%$.

- Evidence suggests an increase in disease prevalence since 1995.

How might it impact on clinical practice in the foreseeable future?

- GERD is likely to remain a common reason for consultation in primary care and referral to secondary care in Europe, North America and other geographic regions, and its prevalence may be increasing. 


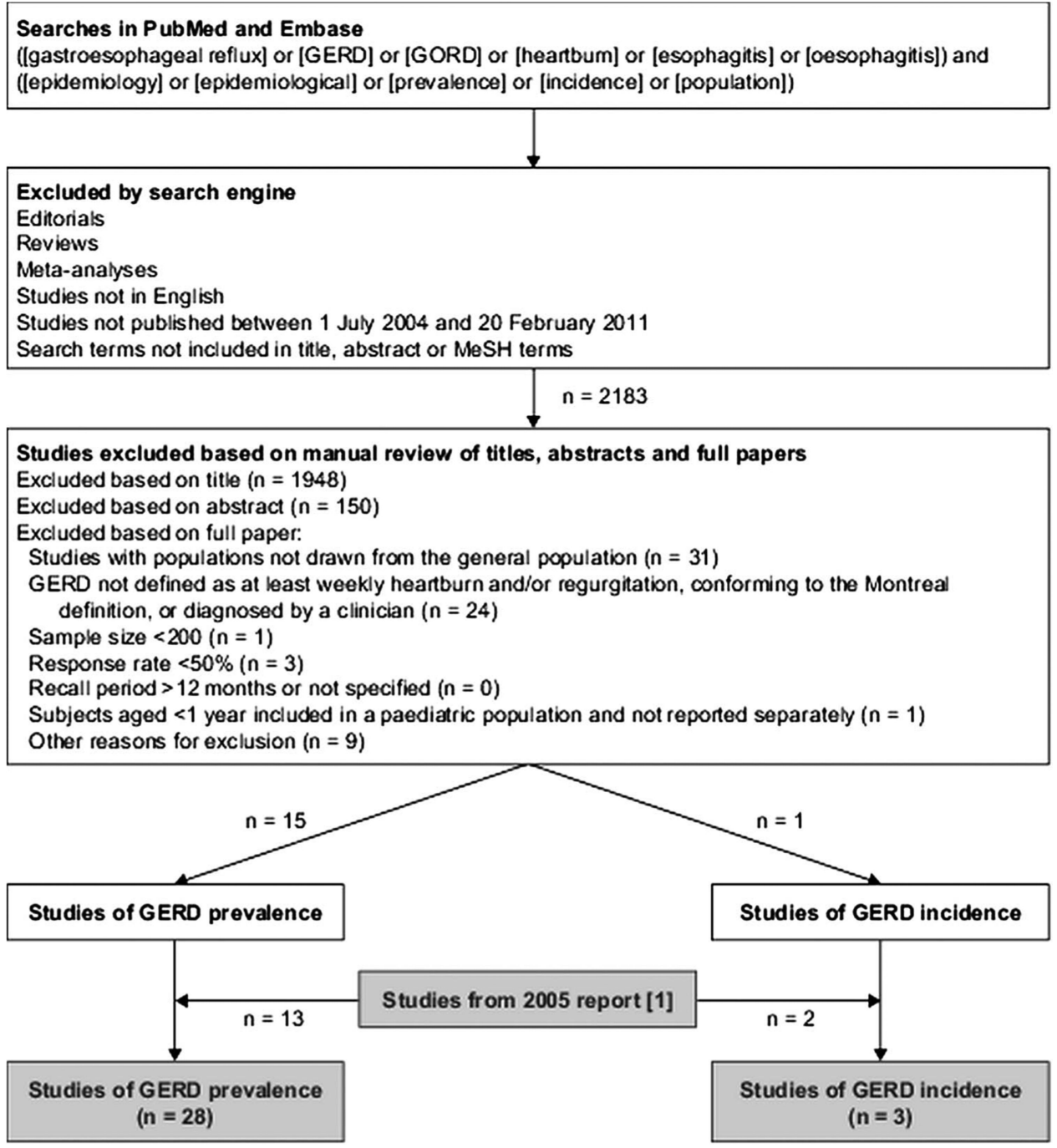

Figure 1.

Flowchart of systematic literature searches. GERD, gastro-oesophageal reflux disease; $\mathrm{MeSH}$, medical subject heading. 


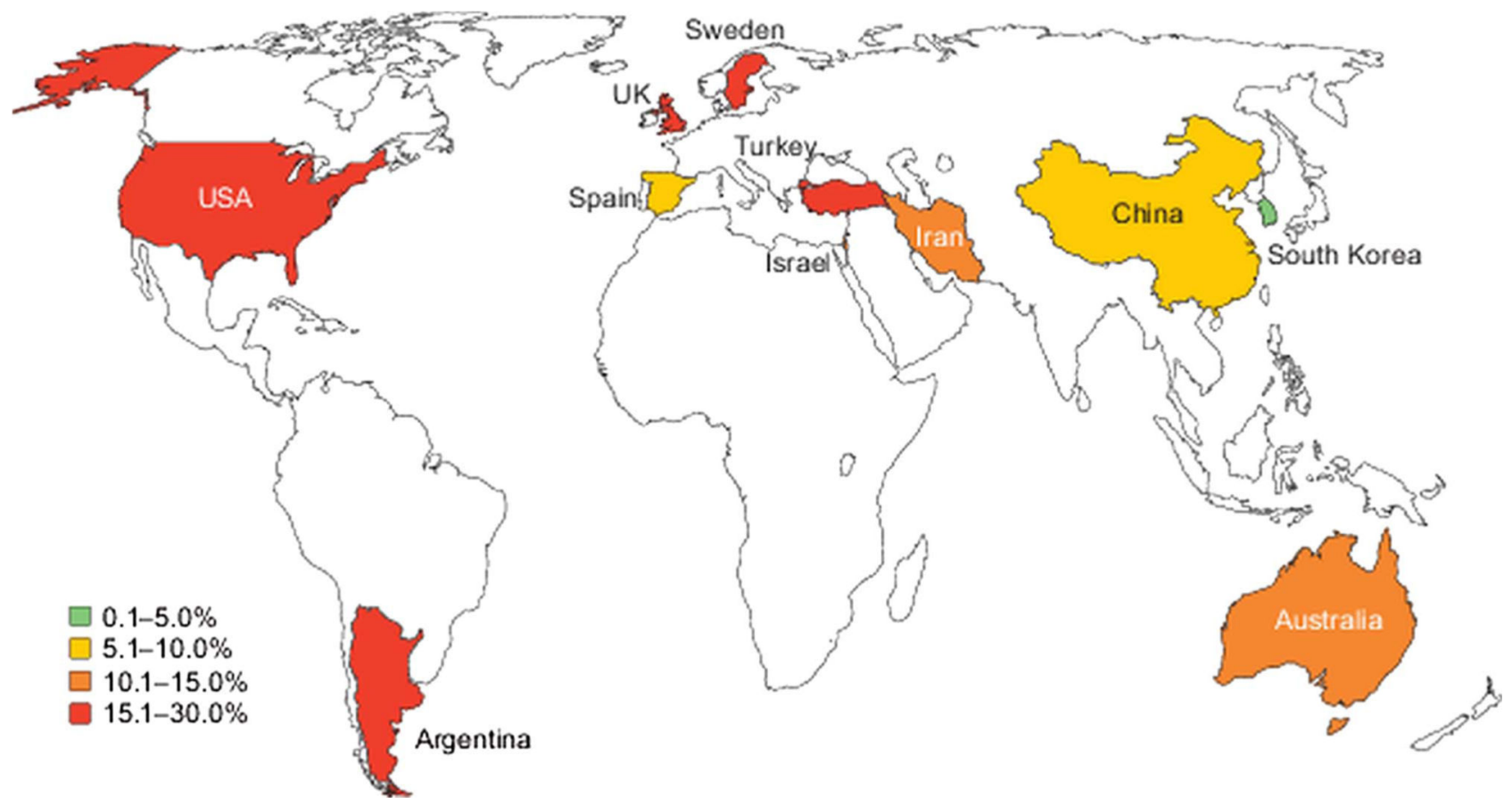

Figure 2.

Global distribution of the burden of gastro-oesophageal reflux disease. Sample-size weighted mean estimates of the prevalence of at least weekly heartburn and/or regurgitation in each country. 


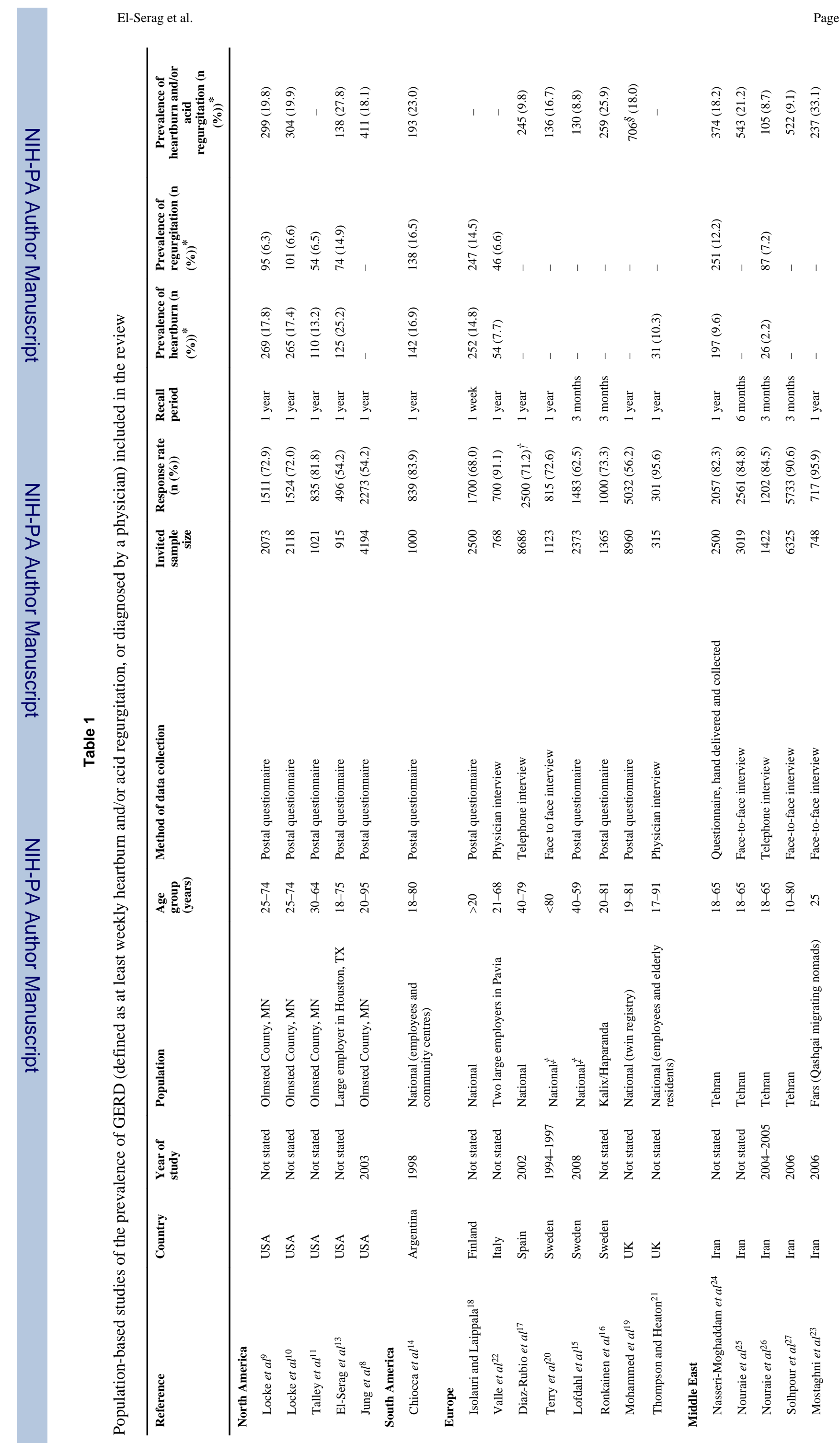




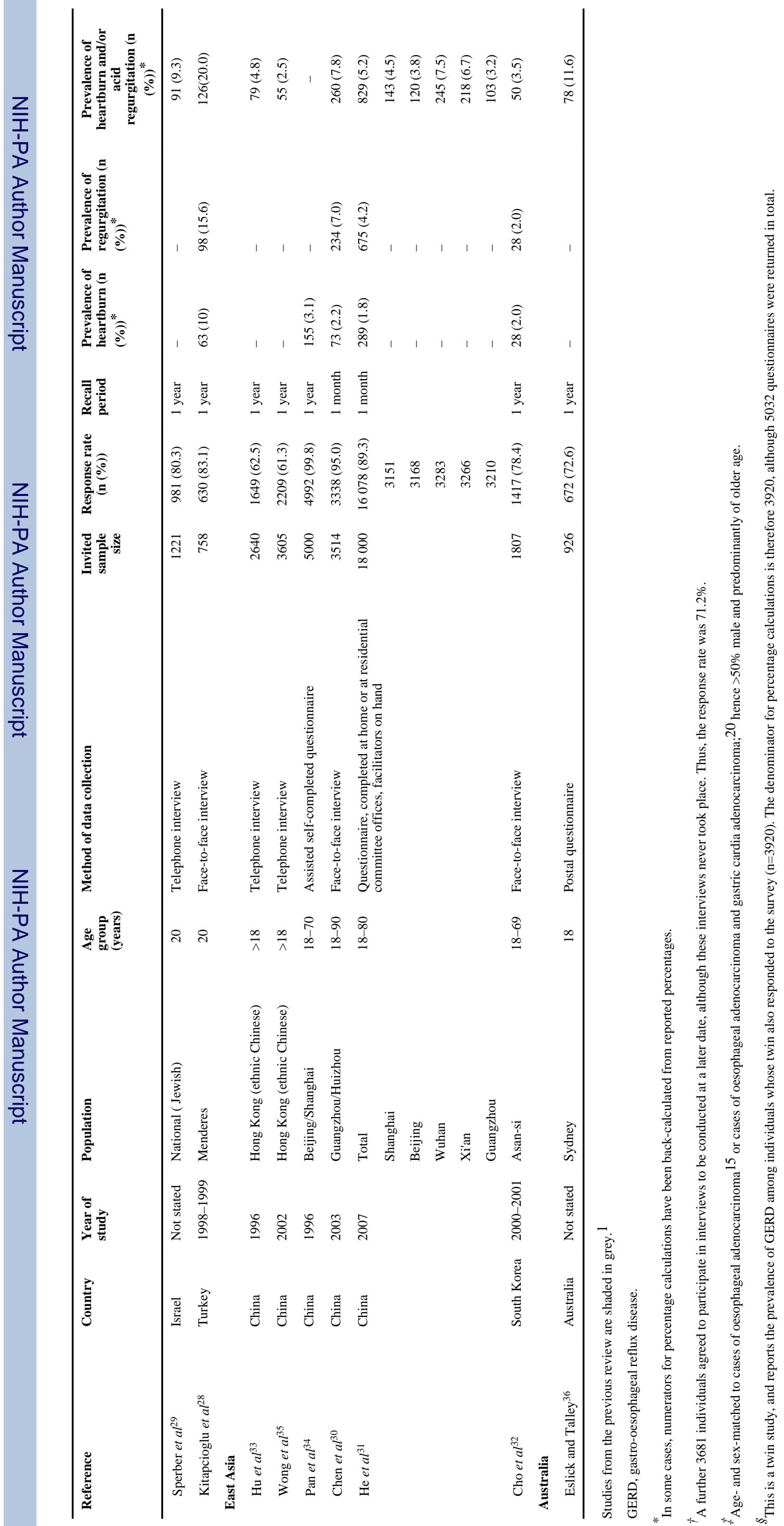


Table 2

Poisson regression analysis of trends in the prevalence of GERD worldwide

\begin{tabular}{|c|c|c|c|c|}
\hline & Included studies & Number of studies & Rate ratio* $(95 \%$ CI $)$ & p Value ${ }^{t}$ \\
\hline \multicolumn{5}{|c|}{ Temporal trends in GERD prevalence } \\
\hline \multicolumn{5}{|l|}{ Study year } \\
\hline Pre-1995 & 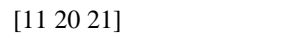 & 3 & Reference & \\
\hline 1995-1999 & {$\left[\begin{array}{llllllll}9 & 10 & 14 & 18 & 22 & 28 & 33 & 34\end{array}\right]$} & 8 & $1.45(1.22$ to 1.73$)$ & $<0.0001$ \\
\hline $2000-2004$ & {$\left[\begin{array}{lllllllll}8 & 13 & 17 & 19 & 26 & 30 & 32 & 35\end{array}\right]$} & 8 & $1.46(1.23$ to 1.74$)$ & $<0.0001$ \\
\hline $2005-2009$ & 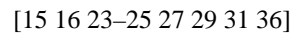 & 9 & $1.51(1.26$ to 1.82$)$ & $<0.0001$ \\
\hline \multicolumn{5}{|c|}{ Geographic trends in GERD prevalence } \\
\hline \multicolumn{5}{|l|}{ Study continent } \\
\hline North America & {$\left[\begin{array}{ll}8-11 & 13\end{array}\right]$} & 5 & Reference & \\
\hline East Asia & [30-35] & 6 & $0.24(0.22$ to 0.26$)$ & $<0.0001$ \\
\hline Europe & [15-22] & 8 & $0.68(0.63$ to 0.73$)$ & $<0.0001$ \\
\hline Middle East & [23-29] & 7 & $0.70(0.64$ to 0.77$)$ & $<0.0001$ \\
\hline
\end{tabular}

Prevalence of at least weekly heartburn used as a surrogate for the prevalence of at least weekly heartburn and/or regurgitation, when no estimate of the prevalence of at least weekly heartburn and/or regurgitation was available.

GERD, gastro-oesophageal reflux disease.

* Assessed using a Poisson regression model, adjusted for geographic region of study conduct.

${ }^{\dagger}$ Assessed using the Wald $\chi^{2}$ test. 
Table 3

Population-based studies of the incidence of GERD.

\begin{tabular}{|c|c|c|c|c|c|c|}
\hline Reference & Country & Data source & Age group (years) & Sample size & $\begin{array}{l}\text { Year of incidence } \\
\text { estimate }\end{array}$ & $\begin{array}{l}\text { Incidence (per } 1000 \\
\text { person-years) }\end{array}$ \\
\hline \multicolumn{7}{|l|}{ USA } \\
\hline Kotzan et $a l^{38}$ & USA & $\begin{array}{l}\text { Georgia Medicaid } \\
\text { claims data }\end{array}$ & $>27$ & 163085 & 1998 & 5.4 \\
\hline \multicolumn{7}{|l|}{ Europe } \\
\hline Ruigómez et al 39 & UK & GPRD & $2-79$ & $\sim 3$ million & 1996 & 4.5 \\
\hline Ruigómez et al ${ }^{37}$ & UK & THIN & $1-17$ & $\sim 2.3$ million & 2000-2005 & 0.84 \\
\hline
\end{tabular}

Studies from the previous review are shaded in grey. 1

GERD, gastro-oesophageal reflux disease; GPRD, General Practice Research Database; THIN, The Health Improvement Network. 